\title{
Autocorrelation Analysis of Particle Magnetization in Erased Particulate Media
}

\author{
M. M. Aziz, B. K. Middleton, and J. J. Miles
}

\begin{abstract}
General analytical expressions for the magnetization power spectral densities of particulate media are derived by using the three-dimensional autocorrelation function. Expressions include the effects of ac demagnetized media and any dc components resulting from the presence of particle chains. The replay flux power spectral density is then obtained, assuming a linear replay transducer.
\end{abstract}

Index Terms-Autocorrelation, magnetization spectrum, noise, particle chaining, particulate media.

\section{INTRODUCTION}

$\mathbf{I}$ N ORDER to predict accurately the measured output spectrum from a magnetic tape, it is important to model closely the state of the particles inside the medium. Expressions for the replay flux spectrum have been produced in the literature by summing the replay flux powers of the individual particles, each with their own random attributes, inside the volume of the medium [1], [2], [3]. As a result, the derived expressions included the contributions of the random aspects of the medium in addition to the well-known head-to-medium replay losses. Work has also been carried out to isolate the terms that are intrinsic to the medium from the general replay flux spectrum [4]. The common facet in these theories was that the particles were assumed independent and to have negligible cross sections in order to simplify the modeling process.

The main emphasis in this paper is on the particle magnetizations in the medium and their statistical behavior. The statistical behavior of the particles is described analytically using the three-dimensional (3-D) spatial autocorrelation function from which the power spectral density can be obtained using the Fourier transformation. For uncorrelated particles, a plausible distribution for particle sizes will be adopted that will produce analytical expressions for the medium spectrum at all wavelengths of interest. Particle chaining will also modeled analytically using the autocorrelation method and the effects of correlations between particles on the medium spectrum will be illustrated. Each vector component of the particle

Manuscript received February 7, 2001; revised August 1, 2001. This work was supported by the Engineering and Physical Sciences Research Council under Grant GR/M46648/01.

M. M. Aziz is with the School of Engineering and Computer Science, University of Exeter, Exeter EX4 4QF, U.K. (e-mail: M.M.Aziz@exeter.ac.uk).

B. K. Middleton is with the Department of Computer Science, University of Manchester, Manchester M13 9PL, U.K. (e-mail: B.Middleton@cs.man.ac.uk).

J. J. Miles is with the Department of Computer Science, University of Manchester, Manchester M13 9PL, U.K. (e-mail: J.Miles@man.ac.uk).

Publisher Item Identifier S 0018-9464(02)00397-7. magnetization power spectral density is then used as the input to the replay head, assumed to act as a linear filter, to predict the output replay flux spectrum.

The analyses will concentrate mainly on a single line of particles taken in the direction of tape motion. Making this line long enough allows the principle of ergodicity to be utilized to make the ensemble averages over all the lines of particles in the volume of the medium to be represented by the spatial average of this single line.

In Section II of this paper, the basic fundamentals regarding the definitions of the spatial autocorrelation function and the statistical moments will be presented. This is followed by the derivation of the autocorrelation function and, therefore, the power spectral density of the particle magnetizations in an array of randomly oriented and independent particles. The effects of particle orientation and length distributions on the medium power spectral density will then be demonstrated analytically. Chaining will be modeled in the following section to explain correlations between particles and the overall power spectral density of the particulate medium is then derived.

In the following derivations, a number of simplifying assumptions are to be made; namely that particles are allowed orientations only in the $x$ and $y$ directions. All the variables in the system including particle lengths, widths, thicknesses and orientations are taken to be independent and therefore uncorrelated. Individual particles and chains are also assumed uncorrelated. The only exception will be in the modeling of particle chaining where the magnetization orientations of adjacent particles in a single chain will be correlated.

\section{DEFINITIONS}

In the case of a real power signal, $q$, the spatial 3-D autocorrelation function can be written as

$$
\begin{array}{r}
R\left(x^{\prime}, y^{\prime}, z^{\prime}\right)=\lim _{V \rightarrow \infty} \frac{1}{V} \iint_{V} \int q(x, y, z) \\
\cdot q\left(x-x^{\prime}, y-y^{\prime}, z-z^{\prime}\right) d x d y d z
\end{array}
$$

where $x^{\prime}, y^{\prime}$ and $z^{\prime}$ are the displacements in the $x, y$ and $z$ directions. If $q$ is bounded in the $y$ and $z$ dimensions and if we are only interested in the wavelength response in the $x$-direction, then the autocorrelation function becomes

$$
\begin{array}{r}
R\left(x^{\prime}, 0,0\right)=\lim _{X \rightarrow \infty} \frac{1}{V} \iint_{V} \int q(x, y, z) \\
\cdot q\left(x-x^{\prime}, y, z\right) d x d y d z .
\end{array}
$$


Since the autocorrelation function is even, the power spectral density in the $x$ direction is given by the Fourier cosine transform of the autocorrelation function [5], $S(k)$

$$
S(k)=2 \int_{x^{\prime}=0}^{\infty} R\left(x^{\prime}, 0,0\right) \cos \left(k x^{\prime}\right) d x^{\prime}
$$

where $k=2 \pi / \lambda$ is the wavenumber and $\lambda$ is the wavelength.

The discrete and continuous versions of the average of a function $f$ of a random variable $r$ are defined respectively as [5]

$$
\begin{aligned}
& \overline{f(r)}=\sum_{i=1}^{\infty} f\left(r_{i}\right) p\left(r_{i}\right) \\
& \overline{f(r)}=\int_{r=-\infty}^{\infty} f(r) p(r) d r
\end{aligned}
$$

where $p(r)$ is the probability density function of the random variable $r$. if

The two random variables $a$ and $b$ are said to be uncorrelated

$$
\overline{a b}=\bar{a} \cdot \bar{b}
$$

and if either variable has a zero mean then the average of their products is zero.

These definitions form the basis of all the derivations carried out in this paper.

\section{UNCORRELATED PARTICLES}

Particles are assumed to have a volume $V^{p}$ with length $l$, width $w$ and thickness $t$ as shown in Fig. 1. Their orientations vary in the $x-y$ plane through an angle $\theta^{p}$ in the range $-\pi / 2$ to $\pi / 2$.

Considering a line of particles of length $L$ in the $x$-direction containing $N$ randomly oriented particles as shown in Fig. 2, then the magnetization along this line can be written as the convolution of an array of unit delta functions, defining the random positions of the particles in the $x$ direction, with the magnetization of a single particle, $M(x, y, z)$

$$
M_{t}(x)=\int_{\tau=-L / 2}^{L / 2} \sum_{i=1}^{N} \alpha_{i} \delta\left(\tau-x_{o}^{i}\right) \cdot M(x-\tau, y, z) d \tau
$$

where $\alpha= \pm 1$ defining the magnetization orientation inside each particle and $x_{o}$ is a random variable defining the positions of individual particles with the origin at the left-hand corner of each particle.

Integration yields

$$
M_{t}(x)=\sum_{i=1}^{N} \alpha_{i} M\left(x-x_{o}^{i}, y, z\right) .
$$

Rewriting $M$

$$
M_{t}(x)=\sum_{i=1}^{N} \mathbf{M}_{\mathbf{i}}^{\mathbf{P}} \alpha_{i} m\left(x-x_{o}^{i}, y, z ; \theta_{i}^{p}\right)
$$

where $m$ is a volume window function of unit magnitude defining the boundaries of each individual particle with orientation $\theta^{p} . \mathbf{M}^{\mathbf{P}}$ is the vector magnetization of individual particles with longitudinal and vertical components $M_{x}^{p}=M^{p} \cos \theta$ and $M_{y}^{p}=M^{p} \sin \theta$ respectively where $M^{p}$ is the saturation magnetization of each particle and $-\pi / 2 \leq \theta \leq \pi / 2$ is the orientation of the magnetization inside each particle.

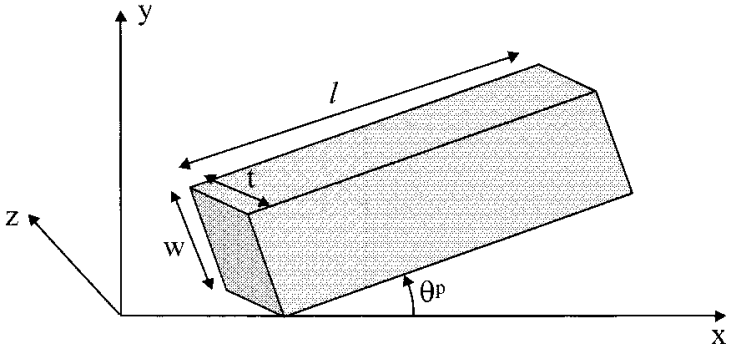

Fig. 1. Geometry and orientation of a single particle.

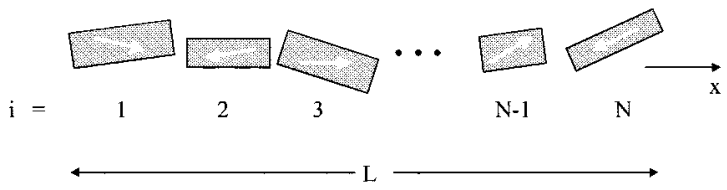

Fig. 2. Line of uncorrelated particles.

Using the definition of (1), the particle magnetization autocorrelation function for this line of particles is given by

$$
\begin{aligned}
R_{M}\left(x^{\prime}, 0,0\right)=\lim _{V_{L} \rightarrow \infty} \frac{1}{V_{L}} \iint_{V_{L}} \int M_{t}(x, y, z) \\
\cdot M_{t}\left(x-x^{\prime}, y, z\right) d x d y d z
\end{aligned}
$$

where $V_{L}$ is the volume of the line of particles. Substitution of (4) into (5) yields the magnetization autocorrelation function as

$$
\begin{aligned}
R_{M}\left(x^{\prime}, 0,0\right) & \\
= & \lim _{V_{L} \rightarrow \infty} \frac{1}{V_{L}} \iint_{V_{L}} \int \sum_{i=1}^{N} \mathbf{M}_{i}^{\mathbf{P}} \alpha_{i} \\
& \cdot m\left(x-x_{o}^{i}, y, z ; \theta_{i}^{p}\right) \\
& \cdot \sum_{j=1}^{N} \mathbf{M}_{j}^{\mathbf{P}} \alpha_{j} \cdot m\left(x-x_{o}^{j}-x^{\prime}, y, z ; \theta_{j}^{p}\right) d x d y d z .
\end{aligned}
$$

Assuming that the particles are uncorrelated and considering the case of an ac erased medium where the net magnetization is zero i.e., $\bar{\alpha}=0$, the cross terms in (6) average to zero and the autocorrelation function becomes

$$
\begin{aligned}
R_{M}\left(x^{\prime}, 0,0\right)=\lim _{V_{L} \rightarrow \infty} \frac{1}{V_{L}} \iint_{V_{L}} \int \sum_{i=1}^{N}\left(\mathbf{M}_{i}^{\mathbf{P}}\right)^{2} \alpha_{i}^{2} \\
\cdot m\left(x, y, z ; \theta_{i}^{p}\right) \\
\cdot m\left(x-x^{\prime}, y, z ; \theta_{i}^{p}\right) d x d y d z
\end{aligned}
$$

where it can be seen that the random variable $x_{o}$ has been dropped without loss of generality as the particles only correlate with themselves. Interchanging the order of the summation and integration and noting that $\alpha_{i}^{2}=1$ leads to

$$
\begin{aligned}
R_{M}\left(x^{\prime}, 0,0\right)=\lim _{V_{L} \rightarrow \infty} \frac{1}{V_{L}} \sum_{i=1}^{N} \iint_{V_{L}} \int\left(\mathbf{M}_{i}^{\mathbf{P}}\right)^{2} \\
\cdot m\left(x, y, z ; \theta_{i}^{p}\right) \\
\cdot m\left(x-x^{\prime}, y, z ; \theta_{i}^{p}\right) d x d y d z .
\end{aligned}
$$


The volume integration is simply given by the area of the shaded region shown in Fig. 3 multiplied by the particle thickness which for a given particle orientation gives

$$
\begin{aligned}
R_{M}\left(x^{\prime}, 0,0\right)=\lim _{V_{L} \rightarrow \infty} & \frac{1}{V_{L}} \sum_{i=1}^{N}\left(\mathbf{M}_{i}^{\mathbf{P}}\right)^{2} t_{i} \\
& \cdot\left(w_{i}-x^{\prime} \sin \theta_{i}^{p}\right)\left(l_{i}-x^{\prime} \cos \theta_{i}^{p}\right) .
\end{aligned}
$$

The power spectral density of the line of particles is simply the Fourier cosine transform of (7) as defined by (2). Before evaluating the power spectral density, the particle correlation length, $u$, for a given particle orientation must first be determined.

This is given by the horizontal dashed line shown in Fig. 4 whose length was found to be

$$
u= \begin{cases}\frac{w}{\sin \left|\theta^{p}\right|} & \left|\theta^{p}\right| \geq \tan ^{-1}\left(\frac{w}{l}\right) \\ \frac{l}{\cos \left|\theta^{p}\right|} & \left|\theta^{p}\right| \leq \tan ^{-1}\left(\frac{w}{l}\right) .\end{cases}
$$

Substituting (7) into (2) and integrating along the limits defined by (8) yields the uncorrelated particle magnetization power spectral density for $\left|\theta^{p}\right| \geq \tan ^{-1}(w / l)$ as

$$
\begin{aligned}
S_{p}(k)= & 2 \lim _{V_{L} \rightarrow \infty} \frac{1}{V_{L}} \sum_{i=1}^{N} \frac{\left(\mathbf{M}_{i}^{\mathbf{P}}\right)^{2} \mathbf{t}_{i}}{k^{2}} \\
& \cdot\left\{w_{i} \cos \left|\theta_{i}^{p}\right|\left(1+\cos \left(\frac{k w_{i}}{\sin \left|\theta_{i}^{p}\right|}\right)\right)\right. \\
& +l_{i} \sin \left|\theta_{i}^{p}\right|\left(1-\cos \left(\frac{k w_{i}}{\sin \left|\theta_{i}^{p}\right|}\right)\right) \\
& \left.-\sin \left(2\left|\theta_{i}^{p}\right|\right) \sin \frac{\left(\frac{k w_{i}}{\sin \left|\theta_{i}^{p}\right|}\right)}{k}\right\} .
\end{aligned}
$$

Noting that

$$
\sum_{i=1}^{N} f\left(x_{i}\right)=N \frac{1}{N} \sum_{i=1}^{N} f\left(x_{i}\right)=N \widetilde{\sim} \sim(x)
$$

where the curly overbar indicates spatial averaging along a single line of particles, then the summation in the power spectral density can be written as an average over all the particles in the line

$$
S_{p}(k)=\frac{2}{k^{2}} \lim _{V_{L} \rightarrow \infty} \frac{N}{V_{L}} \cdot \tilde{\mathbf{M}}^{\mathbf{P})^{2} t}
$$

Assuming ergodicity (i.e., the long spatial average over the particles in a single line is equal to the average of the same line taken over all possible and equally probable, lines of particles in

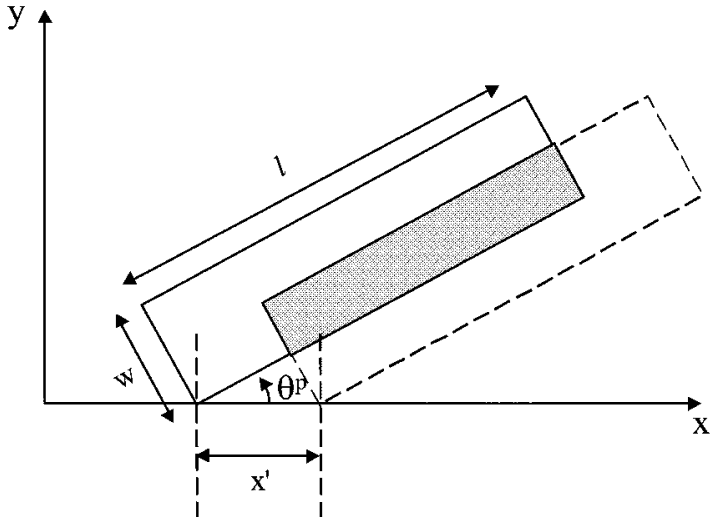

Fig. 3. Calculation of the autocorrelation function of uncorrelated particles.

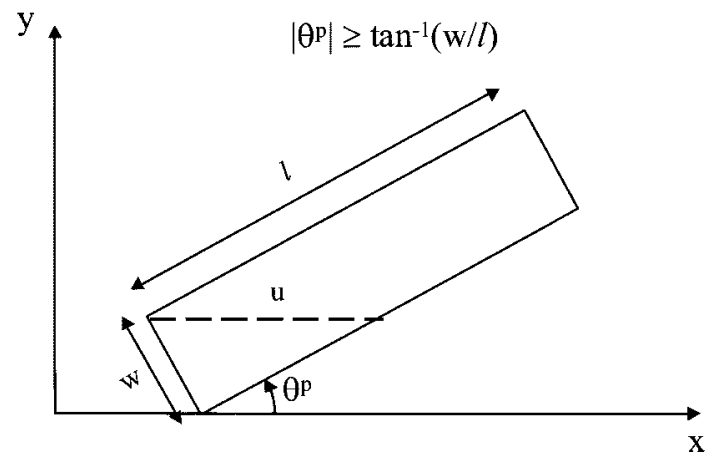

(a)

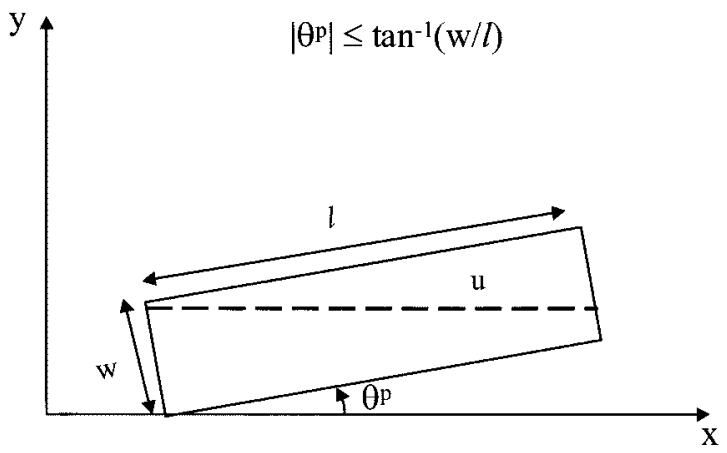

(b)

Fig. 4. Determination of the correlation length.

the volume of the medium under consideration) and noting that $N=\left(P V_{L}\right) / \overline{V^{p}}$ gives

$$
S_{p}(k)=\frac{2 \overline{\left(\mathbf{M}^{\mathbf{P}}\right)^{2}}}{k^{2}} \frac{P}{\overline{V^{p}}} \cdot t\left\{\begin{array}{c}
w \cos \left|\theta^{p}\right|\left(1+\cos \left(\frac{k w}{\sin \left|\theta^{p}\right|}\right)\right) \\
+l \sin \left|\theta^{p}\right|\left(1-\cos \left(\frac{k w}{\sin \mid \theta^{p}}\right)\right) \\
-\sin \left(2\left|\theta^{p}\right|\right) \sin \frac{\left(\frac{k l \mid}{\sin \left|\theta^{p}\right|}\right)}{k}
\end{array}\right\}
$$

and similarly for $\left|\theta^{p}\right| \leq \tan ^{-1}(w / l)$

$$
S_{p}(k)=\frac{2 \overline{\left.\mathbf{M}^{\mathbf{P}}\right)^{2}}}{k^{2}} \frac{P}{\overline{V^{p}}} \cdot t\left\{\begin{array}{c}
l \sin \left|\theta^{p}\right|\left(1+\cos \left(\frac{k l}{\cos \left|\theta^{p}\right|}\right)\right) \\
+w \cos \left|\theta^{p}\right|\left(1-\cos \left(\frac{k l}{\cos \left|\theta^{p}\right|}\right)\right) \\
-\sin \left(2\left|\theta^{p}\right|\right) \sin \frac{\left(\frac{k l}{\cos \left|\theta^{p}\right|}\right)}{k}
\end{array}\right\}
$$


where $\overline{V^{p}}$ is the average volume of an individual particle and $P$ is the volume packing fraction of the lines of particles.

The total power of the particle magnetizations, $P_{M}$, is given by the integral of the particle magnetization power spectral density over the whole range of wavelengths of interest. It is also given by the value of the autocorrelation function with zero displacement in the $x$-direction $\left(x^{\prime}=0\right)$ [5]. According to (7), this is given by

$$
P_{M}=R_{M}(0,0,0)=P \cdot \overline{\left(\mathbf{M}^{\mathbf{P}}\right)^{2}} .
$$

\section{A. Particle Orientation}

It will be assumed that $\theta^{p}$ is independent of the other random variables in the system. For a uniform distribution of particle orientations, it can be shown that for small values of $\left|\theta^{p}\right|<\tan ^{-1}(w / l)$ where $l>w$, (9b) can be written to a very good approximation as

$$
S_{p}(k) \cong \frac{2 \overline{\left(\mathbf{M}^{\mathbf{P}}\right)^{2}}}{k^{2}} \cdot \frac{P}{\overline{V^{p}}} \cdot \overline{t w \cdot(1-\cos (k l))} .
$$

Writing this in terms of the particle density, $D$, where $D=$ $P / \overline{V^{p}}$

$$
S_{p}(k)=\frac{2 \overline{\left(\mathbf{M}^{\mathbf{P}}\right)^{2}}}{k^{2}} \cdot D \cdot \overline{t w \cdot(1-\cos (k l))} .
$$

\section{B. Particle Size Distribution}

In this section, the variations in particle lengths and widths will be assumed to follow the scaled Gamma distribution which for the random variable, $r$, is written as (see the Appendix)

$$
f(r)=\frac{r^{\left(\eta^{2} / \sigma^{2}-1\right)} e^{\left(-r \eta / \sigma^{2}\right)}}{\left(\frac{\sigma^{2}}{\eta}\right)^{\left(\eta^{2} / \sigma^{2}\right)} \Gamma\left(\frac{\eta^{2}}{\sigma^{2}}\right)}
$$

which has mean $\eta$, standard deviation $\sigma$, and whose $n$th moment, evaluated using (3), was found to be

$$
\overline{r^{n}}=\left(\frac{\sigma^{2}}{\eta}\right)^{n} \frac{\Gamma\left(\frac{\eta^{2}}{\sigma^{2}}+n\right)}{\Gamma\left(\frac{\eta^{2}}{\sigma^{2}}\right)}
$$

where $\Gamma(\cdot)$ is the Gamma function. Fig. 5 shows the length distribution of 221 particles obtained from a metal powder sample [6] and compares it with the scaled Gamma distribution of (12) using the measured average particle length and standard deviation. The good agreement between the measured and the calculated distributions justify the utilization of the Gamma distribution to model the variations in particle sizes.

In the following particle lengths, widths and thicknesses are taken to be uncorrelated. For particles with small orientations around the horizontal position, the power spectral density is written from (11) as

$$
S_{p}(k)=\frac{\overline{2\left(\mathbf{M}^{\mathbf{P}}\right)^{2}}}{k^{2}} D \bar{t} \bar{w} \cdot \overline{(1-\cos (k l))}
$$

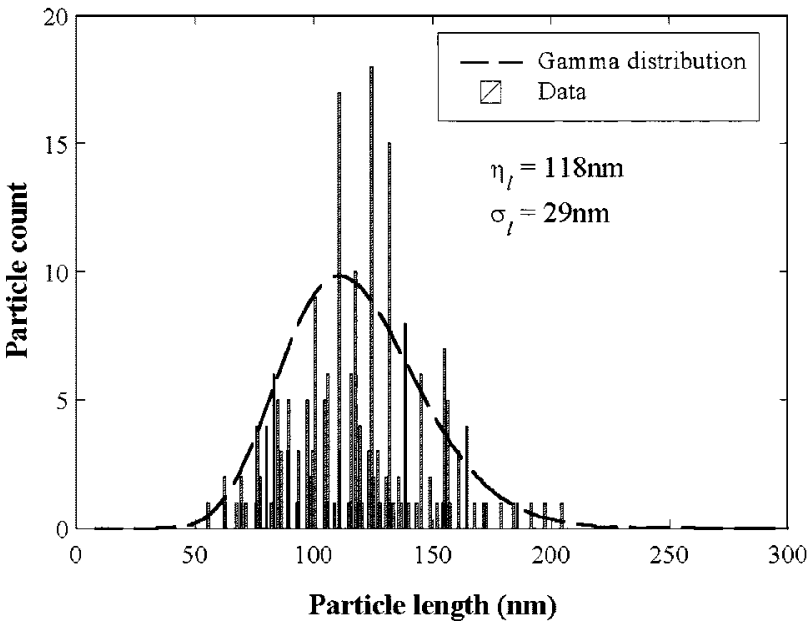

Fig. 5. Experimental and theoretical particle length distributions with mean $118 \mathrm{~nm}$, standard deviation $29 \mathrm{~nm}$ and axial ratio 7.4 [6].

where $\bar{w}$ is the average particle width and $\bar{t}$ is the average particle thickness.

The scaled Gamma distribution will be assumed to apply for particle lengths as well as cross sections. For variations in particle lengths, the average in (14) was evaluated using (3) giving

$$
\overline{1-\cos (k l)}=\left\{1-\frac{\cos \left[\left(\frac{\bar{l}^{2}}{\sigma_{l}^{2}}\right) \tan ^{-1}\left(\frac{k \sigma_{l}^{2}}{\bar{l}}\right)\right]}{\left[1+\left(\frac{k \sigma_{l}^{2}}{\bar{l}}\right)^{2}\right]^{\left(\bar{l}^{2} / \sigma_{l}^{2}\right) / 2}}\right\}
$$

where $\bar{l}$ is the mean particle length and $\sigma_{l}$ is the standard deviation of the particle length distribution. Substituting (15) into (14) yields the particle magnetization power spectral density as

$$
\begin{aligned}
S_{p}(k)=\frac{2 \overline{\left(\mathbf{M}^{\mathbf{P}}\right)^{2}}}{k^{2}} & \cdot D \bar{t} \bar{w} \\
& \cdot\left\{1-\frac{\cos \left[\left(\frac{\bar{l}^{2}}{\sigma_{l}^{2}}\right) \tan ^{-1}\left(\frac{k \sigma_{l}^{2}}{\bar{l}}\right)\right]}{\left[1+\left(\frac{k \sigma_{l}^{2}}{\bar{l}}\right)^{2}\right]^{\left(\bar{l}^{2} / \sigma_{l}^{2}\right) / 2}}\right\} .
\end{aligned}
$$

The longitudinal magnetization component of (16) is plotted in Fig. 6 with emphasis on the particle length distribution effect (wavelength dependent term): this includes an increase of the long wavelength components, a faster rolloff at short wavelengths and the disappearance of the null at wavelengths less than or equal to the average particle length, with the increase in particle length variation.

In the case where $\sigma_{l} \rightarrow 0$, it can be shown that (16) reduces to

$$
S_{p}(k)=\frac{2 \overline{\left(\mathbf{M}^{\mathbf{P}}\right)^{2}}}{k^{2}} \cdot D \bar{w} \bar{t} \cdot(1-\cos (k \bar{l})) .
$$

This is the solid line in Fig. 6. From (16), it can also be seen that the magnetization spectrum is directly proportional to the average particle width and thickness. 


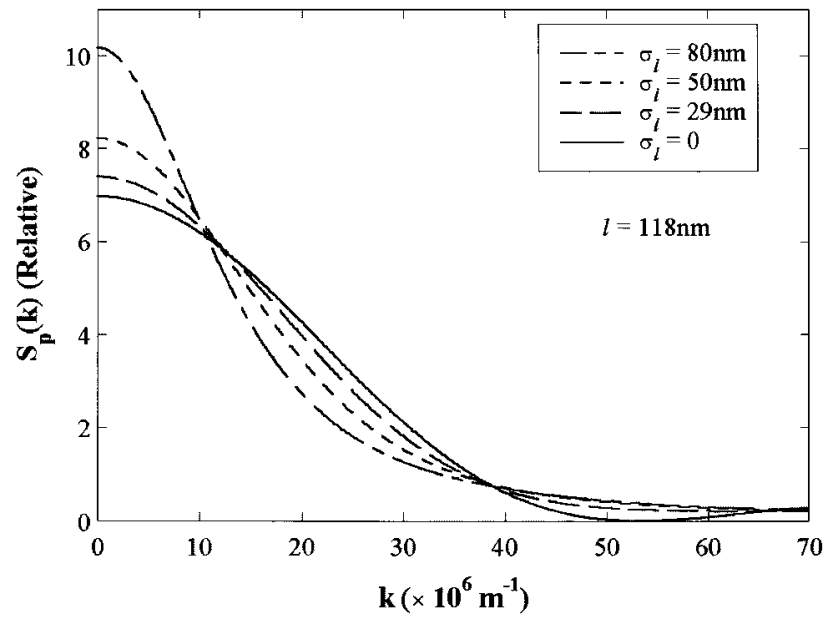

Fig. 6. Effect of particle length distribution on the medium power spectrum.

\section{PARTicle Chaining}

Consider a chain of length $L_{c}$ consisting of $C$ longitudinally oriented and touching particles as shown in two dimensions in Fig. 7.

Working out the spatial 3-D autocorrelation function of the particle magnetizations in a single chain using the shift-multiply-integrate steps as illustrated in the figure yields

$$
\begin{aligned}
& R_{M}^{c}\left(x^{\prime}, 0,0\right)=\sum_{i=1}^{C}\left(\mathbf{M}_{i}^{\mathbf{P}}\right)^{2} \alpha_{i}^{2} t_{i} w_{i}\left(l_{i}-x^{\prime}\right) \\
& \quad+\sum_{i=1}^{C-1}\left(\mathbf{M}_{i}^{\mathbf{P}}\right)^{2} \alpha_{i} \alpha_{i+1}\left[b_{i} t_{i} w_{i}+\left(1-b_{i}\right) t_{i+1} w_{i+1}\right] x^{\prime}
\end{aligned}
$$

where subscripts $i$ and $i+1$ indicate a particle and its nearest neighbor respectively and $b$ is a random variable, equal to 0 or 1 , that allows for the variation of cross-sectional areas of adjacent particles. The first summation accounts for the individual particles in a single chain while the second summation is for the correlation between the adjacent particles in the chain.

Writing the summations as spatial averages in (17)

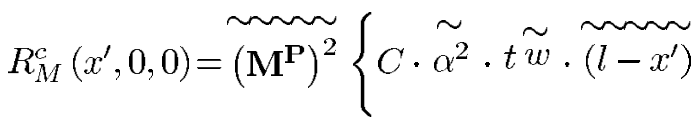

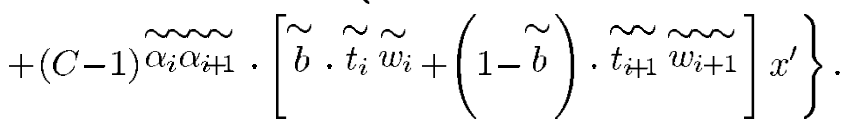

With equal probability of being 1 or 0 (i.e., $p(1)=p(0)=$ $1 / 2$ ), the average of the random variable, $b$, is given by

$$
\widetilde{b}=1 \cdot p(1)+0 \cdot p(0)=\frac{1}{2}
$$

and since on average $\widetilde{w}_{i}=\widetilde{w_{i+1}}$ and $\widetilde{t}_{i}=\widetilde{t_{i+1}}$ the autocorrelation function of a single chain becomes

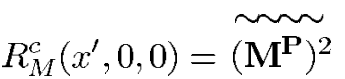

$$
\begin{aligned}
& \tilde{\cdot t \tilde{w}}\left\{\widetilde{\sim} \widetilde{\sim} \widetilde{\sim} \sim \widetilde{\alpha^{2} \cdot\left(l-x^{\prime}\right)+(C-1) \widetilde{\alpha_{i} \alpha_{i+1}} \cdot x^{\prime}}\right\} \text {. }
\end{aligned}
$$

Considering now the line of particles of length $L$ but with $N_{c}$ independent chains, the autocorrelation function becomes

$$
\begin{aligned}
& R_{M}^{c}\left(x^{\prime}, 0,0\right)=\lim _{V_{L} \rightarrow \infty} \frac{1}{V_{L}} \sum_{j=1}^{N_{c}} \sim \sim_{\left.\mathbf{M}^{\mathbf{P}}\right)_{j}^{2}}^{\sim} \tilde{t_{j}} \sim \tilde{w}_{j}
\end{aligned}
$$

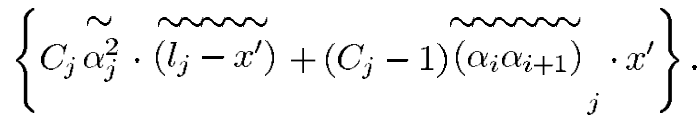

Since the chains are uncorrelated, the correlation length will effectively be the length $L_{c}$ of a single chain, i.e.,

$$
L_{c}=\sum_{i=1}^{C} l_{i}=C \tilde{l}
$$

where $\tilde{l}$ is the average particle length in a single chain. Taking the Fourier cosine transform of (18) along the chain length, $L_{c}$ and assuming ergodicity, gives the magnetization power spectral density of the chains in the line of particles as

$S_{c}(k)$

$$
\begin{aligned}
= & \frac{2 \overline{\left(\mathbf{M}^{\mathbf{P}}\right)^{2}}}{k^{2}} \cdot \bar{t} \bar{w} \cdot \lim _{V_{L} \rightarrow \infty} \frac{N_{c}}{V_{L}} \\
& \cdot\left\{\overline{\alpha^{2}} \cdot \overline{C[1-\cos (k C \bar{l})-(C-1) k \bar{l} \sin (k C \bar{l})]}\right. \\
& -\overline{\alpha_{i} \alpha_{i+1}} \cdot \overline{(C-1)[1-\cos (k C \bar{l})-k C \bar{l} \sin (k C \bar{l})]} .
\end{aligned}
$$

Letting the total number of particles in the line be $N$, such that, $V_{L}=N_{v^{p}} / P$, the power spectral density becomes $S_{c}(k)$

$$
\begin{aligned}
= & \frac{2 \overline{\left(\mathbf{M}^{\mathbf{P}}\right)^{2}}}{k^{2}} \cdot \bar{t} \bar{w} \cdot \lim _{N \rightarrow \infty} \frac{N_{c} P}{N \overline{v^{p}}} \\
& \cdot\left\{\overline{\alpha^{2}} \cdot \overline{C[1-\cos (k C \bar{l})-(C-1) k \bar{l} \sin (k C \bar{l})]}\right. \\
& \left.-\overline{\alpha_{i} \alpha_{i+1}} \cdot \overline{(C-1)[1-\cos (k C \bar{l})-k C \bar{l} \sin (k C \bar{l})]}\right\} .
\end{aligned}
$$

Adding the contribution of the remaining $\left(N-N_{c} \bar{C}\right)$ uncorrelated particles in this line of particles and writing in terms of the particle density yields

$S(k)$

$$
\begin{aligned}
= & \frac{\overline{\left(\mathbf{M}^{\mathbf{P}}\right)^{2}}}{k^{2}} \cdot \bar{t} \bar{w} \cdot \lim _{N \rightarrow \infty} \frac{D}{N}\left\{\left(N-N_{c} \bar{C}\right) \cdot \overline{(1-\cos (k l))}\right. \\
& +N_{c} \cdot \overline{\alpha^{2}} \cdot \overline{C[1-\cos (k C \bar{l})-(C-1) k \bar{l} \sin (k C \bar{l})]} \\
& \left.-N_{c} \cdot \overline{\alpha_{i} \alpha_{i+1}} \cdot \overline{(C-1)[1-\cos (k C \bar{l})-k C \bar{l} \sin (k C \bar{l})]}\right\} .
\end{aligned}
$$

If we write $\lim _{N \rightarrow \infty} N_{c} / N=F$, i.e., the probability of finding a chain in $N$ particles, then

$S(k)$

$$
\begin{aligned}
= & \frac{2 \overline{\left(\mathbf{M}^{\mathbf{P}}\right)^{2}}}{k^{2}} \cdot D \bar{t} \bar{w} \cdot\{(1-F \bar{C}) \cdot \overline{(1-\cos (k l))} \\
& +F \cdot \overline{\alpha^{2}} \cdot \overline{C[1-\cos (k C \bar{l})-(C-1) k \bar{l} \sin (k C \bar{l})]} \\
& \left.-F \cdot \overline{\alpha_{i} \alpha_{i+1}} \cdot \overline{(C-1)[1-\cos (k C \bar{l})-k C \bar{l} \sin (k C \bar{l})]}\right\}
\end{aligned}
$$

where $0 \leq F \leq 1$. 


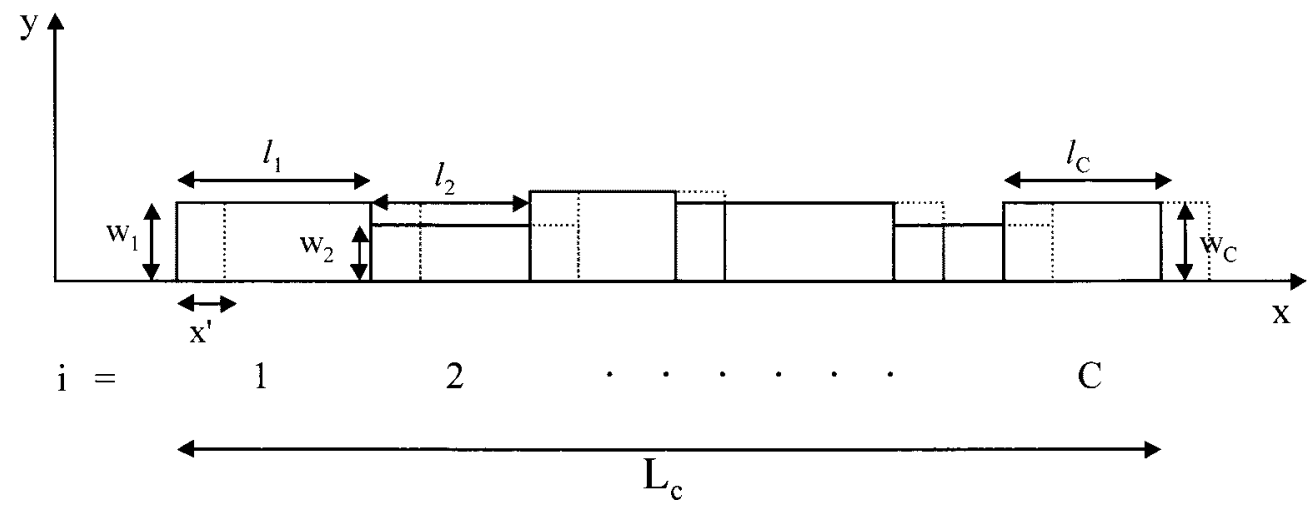

Fig. 7. A chain of particles.

It can be seen from (19) that in the case where $C=1$ the magnetization power spectral density reduces to the case of uncorrelated particles. Furthermore, when $F=0$, i.e., no chains, then the power spectral density is, again, that of an assembly of uncorrelated particles as given by (14).

\section{A. Magnetization Orientation Cross Correlation}

In (19) $\overline{\alpha^{2}}=1$. On the other hand, the cross-correlation term in (19) can be written as

$$
\overline{\alpha_{i} \alpha_{i+1}}=\overline{\alpha_{i} \alpha_{j}} \quad j=i+1 .
$$

Evaluating the average by counting all the probabilities of the magnetization orientations gives

$$
\begin{aligned}
\overline{\alpha_{i} \alpha_{j}}= & (+1)(+1) P_{i j}(+1,+1)+(-1)(-1) P_{i j}(-1,-1) \\
& +(-1)(+1) P_{i j}(-1,+1)+(+1)(-1) P_{i j}(+1,-1)
\end{aligned}
$$

where $P_{i j}$ is the joint probability density of two adjacent particle magnetization spins. By symmetry

$$
\overline{\alpha_{i} \alpha_{j}}=2 P_{i j}(1,1)-2 P_{i j}(1,-1) .
$$

Using Bayes' rule [5], the joint probabilities can be written in terms of conditional probabilities as

$$
\overline{\alpha_{i} \alpha_{j}}=2\left[P_{i \mid j}(1 \mid 1) P_{j}(1)-P_{i \mid j}(1 \mid-1) P_{j}(-1)\right]
$$

where $P_{i \mid j}(1 \mid 1)$ is the probability of $i$ being 1 given that $j$ is 1 and $P_{i \mid j}(1 \mid-1)$ is the probability of $i$ being 1 given that $j$ is -1 .

Since 1 and -1 can occur with equal probability

$$
P(1)=P(-1)=\frac{1}{2}
$$

and

$$
P_{i \mid j}(1 \mid 1)=1-P_{i \mid j}(1 \mid-1)
$$

and so the cross-correlation term becomes

$$
\overline{\alpha_{i} \alpha_{j}}=1-2 P_{i \mid j}(1 \mid-1) .
$$

Writing

$$
P_{t}\left(\begin{array}{c}
\text { probability of a transition } \\
\text { between two adjacent particles }
\end{array}\right)=P_{i \mid j}(1 \mid-1)
$$

then

$$
\overline{\alpha_{i} \alpha_{j}}=1-2 P_{t} \quad 0 \leq P_{t} \leq 1
$$

and substituting into (19) and simplifying yields the magnetization power spectral density

$$
\begin{aligned}
& S(k)=\frac{2 \overline{\left(\mathbf{M}^{\mathbf{P}}\right)^{2}}}{k^{2}} \cdot D \bar{t} \bar{w} \\
& \text { - }\{(1-F \bar{C}) \cdot \overline{(1-\cos (k l))} \\
& +2 F P_{t}[\bar{C}-\overline{C \cos (k C \bar{l})} \\
& -k \bar{l} \cdot \overline{C^{2} \sin (k C \bar{l})} \\
& +k \bar{l} \cdot \overline{C \sin (k C \bar{l})}] \\
& \left.+F\left(1-2 P_{t}\right)[1-\overline{\cos (k C \bar{l})}]\right\} \text {. }
\end{aligned}
$$

\section{B. Chain Length Distribution}

If the chain lengths $C$ in (21) were allowed to take discrete values between 1 and a maximum of $C_{1}$, inclusive, with a uniform density function $1 / C_{1}$, then the averages in (21) can be worked out analytically using (3).

Using the identities [10]

$$
\begin{gathered}
\sin (\psi)+\sin (2 \psi)+\sin (3 \psi)+\cdots+\sin (n \psi) \\
=\frac{\sin \left(\frac{\psi(n+1)}{2}\right) \sin \left(\frac{n \psi}{2}\right)}{\sin \left(\frac{\psi}{2}\right)}
\end{gathered}
$$

and:

$$
\begin{gathered}
\cos (\psi)+\cos (2 \psi)+\cos (3 \psi)+\cdots+\cos (n \psi) \\
=\frac{\cos \left(\frac{\psi(n+1)}{2}\right) \sin \left(\frac{n \psi}{2}\right)}{\sin \left(\frac{\psi}{2}\right)}
\end{gathered}
$$

and their appropriate derivatives with respect to $\psi$, the averages in (21) were found to be

$$
\bar{C}=\frac{\left(C_{1}+1\right)}{2}
$$




\section{$\overline{C \cos (k C \bar{l})}$}

$$
\begin{aligned}
= & \frac{\left(C_{1}+1\right) \cos \left(\frac{k \bar{l}\left(C_{1}+1\right)}{2}\right) \sin \left(\frac{k C_{1} \bar{l}}{2}\right)}{2 C_{1} \sin \left(\frac{k \bar{l}}{2}\right)} \\
& +\frac{\sin \left(\frac{k \bar{l}\left(C_{1}+1\right)}{2}\right)}{2 C_{1} \sin \left(\frac{k \bar{l}}{2}\right)}\left[C_{1} \cos \left(\frac{k C_{1} \bar{l}}{2}\right)\right. \\
& \left.-\frac{\sin \left(\frac{k C_{1} \bar{l}}{2}\right) \cos \left(\frac{k \bar{l}}{2}\right)}{\sin \left(\frac{k \bar{l}}{2}\right)}\right]
\end{aligned}
$$

$\overline{C^{2} \sin (k C \bar{l})}$

$$
\begin{aligned}
= & \frac{1}{8 C_{1} \sin ^{3}\left(\frac{k \bar{l}}{2}\right)}\left[C_{1}^{2} \cos \left(\frac{k \bar{l}}{2}\left(2 C_{1}+3\right)\right)\right. \\
& -2 \cos \left(\frac{k \bar{l}}{2}\right)+\left(C_{1}+1\right)^{2} \cos \left(\frac{k \bar{l}}{2}\left(1-2 C_{1}\right)\right) \\
& \left.+\left(1-2 C_{1}\left(C_{1}+1\right)\right) \cos \left(\frac{k \bar{l}}{2}\left(1+2 C_{1}\right)\right)\right](22 \mathrm{c})
\end{aligned}
$$

$\overline{C \sin (k C \bar{l})}$

$$
=\frac{\left(C_{1}+1\right) \sin \left(k C_{1} \bar{l}\right)-C_{1} \sin \left(k \bar{l}\left(C_{1}+1\right)\right)}{4 C_{1} \sin ^{2}\left(\frac{k \bar{l}}{2}\right)}
$$

$\overline{\cos (k C \bar{l})}$

$$
=\frac{\cos \left(\frac{k \bar{l}\left(C_{1}+1\right)}{2}\right) \sin \left(\frac{k C_{1} \bar{l}}{2}\right)}{C_{1} \sin \left(\frac{k \cdot \bar{l}}{2}\right)} .
$$

Substituting the averages from (15) and (22) into (21), the effects of $F$ (chains percentage), $C$ (chain length) and $P_{t}$ (correlation strength) on the magnetization spectrum can now be investigated.

In Fig. 8, the particle magnetization power spectral density is plotted for different values of $F$ for an average particle length of $118 \mathrm{~nm}$ and standard deviation $29 \mathrm{~nm}$. From the figure, it can be seen that increasing the percentage of chains in the medium enhances the magnetization spectrum at long wavelengths and causes an attenuation of the spectrum at shorter wavelengths.

Increasing the chain length, on the other hand, leads to an enhancement of the spectrum that is more confined to long wavelengths with less attenuation at short wavelengths for the same particle length and standard deviation as demonstrated in Fig. 9.

Reducing the correlation between the adjacent particle magnetization spins (increasing $P_{t}$ ) in each chain reduces the dc content of the magnetization spectrum as shown in Fig. 10. Since the particles in each chain are contiguous, reducing the magnetization spin correlations also causes discontinuities in the magnetization of each chain and, hence, ripples in the calculated spectrum.

\section{REPLAY FluX POWER SPECTRAL DENSITy}

If the replay process is assumed linear then the replay flux power spectral density for a single line of particles is the product

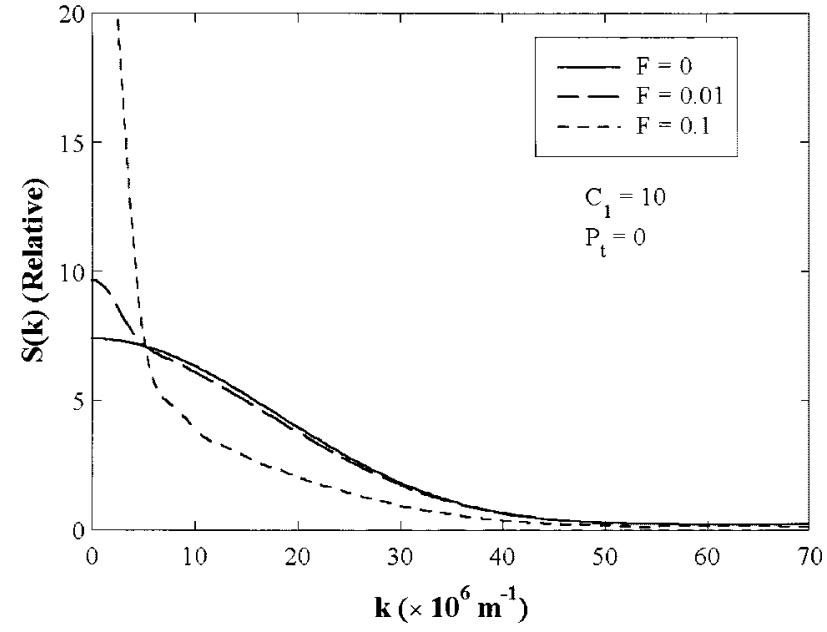

Fig. 8. Effect of increasing the precentage of chains $(F)$ in the medium on the magnetization power spectral density.

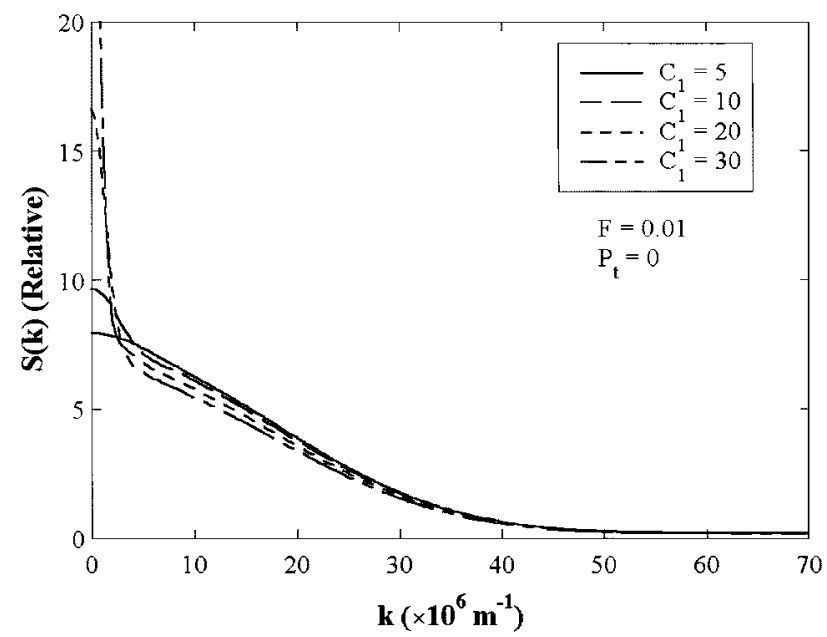

Fig. 9. Effect of the maximum chain length $\left(C_{1}\right)$ on the particle magnetization power spectral density.

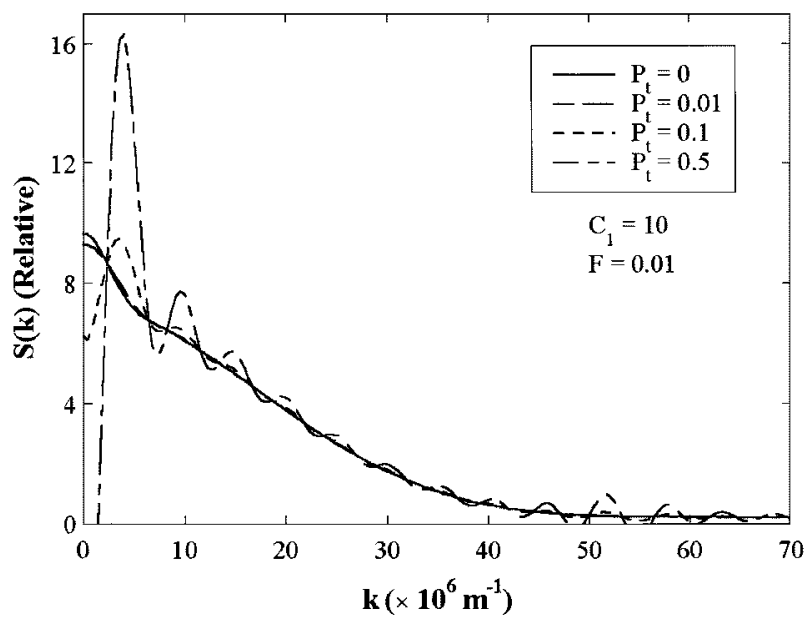

Fig. 10. Effect of reducing the particle correlations (increasing $P_{t}$-the probability of switching the magnetization between two adjacent particles) on the magnetization spectrum. 
of the particle magnetization power spectral density and the squared magnitude of the replay head transfer function [5]

$$
S_{\Phi}(k)=\left|\mu_{0} \frac{\mathbf{H}(k)}{I}\right|^{2} S(k)
$$

where $\mathbf{H}(k)$ is the Fourier transform of the head field function or sensitivity function and contains the contributions of the longitudinal and vertical components. Adding the longitudinal and vertical components of medium magnetization and head spectra

$$
\begin{aligned}
S_{\Phi}(k)= & \left(\frac{\mu_{0}}{I}\right)^{2} \\
& \cdot\left[\left|H_{x}(k)\right|^{2} S(k) \cdot \overline{\cos ^{2} \theta}+\left|H_{y}(k)\right|^{2} S(k) \cdot \overline{\sin ^{2} \theta}\right] .
\end{aligned}
$$

Since the two-dimensional head field components are a Hilbert transform pair [3], the replay flux power spectral density becomes

$$
S_{\Phi}(k)=\left(\frac{\mu_{0}}{I}\right)^{2}\left|H_{x}(k)\right|^{2} S(k) \cdot\left[\overline{\cos ^{2} \theta}+\overline{\sin ^{2} \theta}\right]
$$

where $H_{x}(k)$ is the longitudinal field transform and can be written in terms of the surface field transform (or gap loss function), $H_{s}(k)$, times the exponential spacing loss factor as [7]

$$
H_{x}(k)=H_{s}(k) \cdot e^{-k y} .
$$

Substituting into (23) yields the replay flux spectrum as

$$
S_{\Phi}(k)=\left(\frac{\mu_{0}}{I}\right)^{2}\left|H_{s}(k)\right|^{2} e^{-2 k y} \cdot S(k) \cdot\left[\overline{\cos ^{2} \theta}+\overline{\sin ^{2} \theta}\right] .
$$

Including all the lines of particles across the track, $W$ and through the thickness of the medium, $\delta$ (assuming that these dimensions are large compared to the particle cross section) yields

$$
\begin{array}{r}
S_{\Phi}(k)=\int_{z=0}^{W} \int_{y=d}^{d+\delta}\left(\frac{\mu_{0}}{I}\right)^{2}\left|H_{s}(k)\right|^{2} e^{-2 k y} \cdot S(k) \\
\cdot\left[\overline{\cos ^{2} \theta}+\overline{\sin ^{2} \theta}\right] d y d z
\end{array}
$$

where $d$ is the replay head-to-medium separation. Assuming that the track width is much larger than the average particle thickness and integrating through the depth yields

$$
\begin{aligned}
& S_{\Phi}(k)=W\left(\frac{\mu_{o}}{I}\right)^{2}\left|H_{s}(k)\right|^{2} S(k) \\
& \cdot e^{-2 k \cdot d}\left(\frac{1-e^{-2 k d}}{k}\right) \cdot\left[\overline{\cos ^{2} \theta}+\overline{\sin ^{2} \theta}\right] .
\end{aligned}
$$

If $\theta$ (magnetization orientation inside each particle) is allowed to vary uniformly about the horizontal position, then it can be shown that

$$
\left[\overline{\cos ^{2} \theta}+\overline{\sin ^{2} \theta}\right]=1
$$

and the flux power spectral density reduces to [3]

$$
S_{\Phi}(k)=W\left(\frac{\mu_{0}}{I}\right)^{2}\left|H_{s}(k)\right|^{2} S(k) \cdot e^{-2 k d}\left(\frac{1-e^{-2 k d}}{k}\right) .
$$

The replay voltage for an inductive head is simply the time derivative of the replay flux. The voltage power spectral density, $S_{E}(k)$, can then be obtained from (25) by multiplying by the square of the head-to-medium velocity and the wavenumber, i.e.,

$$
S_{E}(k)=v^{2}|k|^{2} S_{\Phi}(k) .
$$

\section{DISCUSSION}

The autocorrelation method was used to model the statistical behavior of an ac erased or a virgin particulate medium containing individual, uncorrelated, particles and chains of particles with controlled interactions. Using this method allowed the derivation of a general expression of the magnetization power spectral density of a particulate medium taking into account the particle size, orientation, and correlation between adjacent particles independently of the replay transducer. This expression, equation (21), can also be used to represent the "background noise" present in tape systems due to the particulate nature of the recording medium.

By modeling the variance of particle sizes with the Gamma distribution, it was possible to obtain an analytical expression for the magnetization power spectral density of uncorrelated particles for all wavelengths of interest as indicated in Fig. 6 . It is interesting to note that the derived expression and the calculated spectra produced using this plausible distribution of particle sizes are in a similar form to those found in [4] (from simple modeling of particle chaining) and [8] (by assuming correlations between particle lengths and widths).

Equation (21) for the magnetization power spectral density of the medium is controlled by three factors; namely $F$ (the percentage of chains), $C_{1}$ (the maximum chain length) and $P_{t}$ (the probability of switching between two adjacent particles). By setting $F=0$ in (21), for example, the spectrum reduces to that of individual and uncorrelated particles. With $P_{t}=0$, (21) produce the magnetization spectrum of a medium containing individual and chains of strongly coupled, contiguous, particles with uniform magnetization. Reducing the coupling between the particles is achieved with values of $P_{t}>0$ which produces magnetization spectrum with ripples. Experimental measurements on an advanced helical scan tape system using high coercivity metal particle tapes have been carried out and fittings to the derived spectra have been produced. Good agreement with measurements was obtained and the experimental measurements and fittings will be reported in future publications.

\section{CONCLUSION}

General expressions for the magnetization power spectral densities that model the state of the particles in tape media and predict the observed spectra were presented. In addition to predicting the behavior of an assembly of uncorrelated particles, the effect of correlations between particles was also modeled analytically. Correlations between particle magnetizations were found to contribute an enhancement of the magnitude of the medium spectrum at long wavelengths. 


\section{APPENDIX}

The Gamma distribution for the random variable $r$ with parameters $\beta$ and $\gamma$ is given by [9]

$$
f(r)=\frac{1}{\gamma^{\beta} \Gamma(\beta)} \cdot r^{\beta-1} e^{-r / \gamma}
$$

with mean $\eta=\gamma \beta$, standard deviation $\sigma=\sqrt{\beta} \gamma$ and where

$$
\int_{r=0}^{\infty} f(r) d r=1
$$

Solving the mean and standard deviation above for $\beta$ and $\gamma$ yields

$$
\beta=\frac{\eta^{2}}{\sigma^{2}} \quad \text { and } \quad \gamma=\frac{\sigma^{2}}{\eta} .
$$

Substituting back yields the scaled Gamma distribution

$$
f(r)=\frac{r^{\left(\eta^{2} / \sigma^{2}-1\right)} e^{\left(-r \eta / \sigma^{2}\right)}}{\left(\frac{\sigma^{2}}{\eta}\right)^{\left(\eta^{2} / \sigma^{2}\right)} \Gamma\left(\frac{\eta^{2}}{\sigma^{2}}\right)}
$$

with mean $\eta$ and standard deviation $\sigma$.

\section{ACKNOWLEDGMENT}

The authors are in debt to M. del Puerto Morales Herrero of the Materials Science Institute of Madrid for providing the data of the particle length counts.

\section{REFERENCES}

[1] I. Stein, "Analysis of noise from magnetic storage media," J. Appl. Phys., vol. 34, no. 7, pp. 1976-1990, 1963.

[2] L. Thurlings, "Statistical analysis of signal and noise in magnetic recording," IEEE Trans. Magn., vol. MAG-16, pp. 507-513, 1980.

[3] H. N. Bertram, Theory of Magnetic Recording. Cambridge, U.K.: Cambridge Univ. Press, 1994.

[4] L. L. Nunnelley, D. E. Heim, and T. C. Arnoldussen, "Flux noise in particulate media: Measurement and interpretation," IEEE Trans. Magn., vol. MAG-23, pp. 1767-1775, Sept. 1987.

[5] B. P. Lathi, Modern Digital and Analog Communication Systems, 3rd ed, New York: Oxford Univ. Press, 1998.

[6] M. P. Morales, S. A. Walton, L. S. Prichard, C. J. Serna, D. P. E. Dickson, and K. O'Grady, "Characterization of advanced metal particle recording media pigments," J. Magn. Magn. Mater, vol. 190, pp. 357-370, 1998.

[7] D. A. Lindholm, "Dependence of reproducing gap null on head geometry," IEEE Trans. Magn., vol. 11, no. 6, pp. 1692-1696, 1975.

[8] D. Denteneer and H. Cramer, "Correlation of particle length and radius: A new model for flux noise in particulate media," IEEE Trans. Magn., vol. 26, pp. 2110-2112, Sept. 1990.

[9] CRC Handbook of Mathematical Sciences, 6th ed., W. H., Ed., CRC Press, Boca Raton, FL, 1987.

[10] H. B. Dwight, Tables of Integrals and Other Mathematical Data, 4th ed. Ontario, Canada: Macmillan, 1961. 